\title{
Pacemaker Twiddler Syndrome - A Rare but Serious Cause of Pacemaker Malfunction
}

\author{
Gurkirat Singh*, Hemant Khemani, Zahidullah Khan and Narender Omprakash Bansal \\ Department of Cardiology, Grant Medical College and Sir JJ Group of Hospitals, Mumbai, India
}

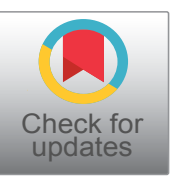

*Corresponding author: Gurkirat Singh, Department of Cardiology, Grant Medical College and Sir JJ Group of Hospitals, Byculla, Mumbai-400008, India, Tel: $+91-9833826672$

\begin{abstract}
Twiddler's syndrome is a rare complication after pacemaker implantation. It is caused by conscious or unconscious manipulation at the implantation site by the patient with the result of device malfunction. It results in lead dislodgment, diaphragmatic stimulation, and loss of capture. In a pacemaker dependent patient, it can be a lethal complication. It usually occurs in the first year following pacemaker implantation. The treatment involves readmission, repositioning of the dislodged leads and suture fixation of the lead and pulse generator within its pocket. We report a case of 84-year-old female who was readmitted after 4 weeks of implantation of permanent pacemaker with syncope and diaphragmatic stimulation.
\end{abstract}

\section{Keywords}

Twiddler's syndrome, Lead dislodgement, Pacemaker malfunction

\section{Introduction}

Twiddler's syndrome was first described by Bayliss, et al. in 1968, when a patient manipulated and rotated the pulse generator in the pocket resulting in lead dislodgement, diaphragmatic stimulation, and loss of capture [1]. It occurs because of unintentional or deliberate manipulation of the pulse generator within its skin pocket by the patient. This results in coiling of the pacemaker lead due to the rotation of pacemaker generator on its long axis. The coiling of the lead results in lead retraction, lead damage and cause lead fracture or insulation leakage. We report a case of 84-year-old female who presented with syncope and twitching sensation in the right upper quadrant of the abdomen, 4 weeks after the implantation of a permanent pacemaker.

\section{Case Report}

84-year-old female was admitted with recurrent syncope. She is a known diabetic and hypertensive. She is also a case of the chronic obstructive pulmonary disease. On admission pulse was 34/minute and blood pressure was $160 / 90 \mathrm{mmHg}$. Electrocardiogram showed atrial fibrillation with a ventricular rate of 34 /minute. Temporary pacemaker lead was inserted through the right femoral venous route. All the reversible causes of the condition were ruled out. Echocardiography showed mild dilatation of the left atrium, mild concentric left ventricular hypertrophy with an ejection fraction of $60 \%$. A Single chamber permanent pacemaker (ST. JUDE -VVI) was implanted in the right infra-clavicular area. A bipolar active fixation, screwing lead was placed at the right ventricular apex. The pulse generator was also fixed to the underlying pectoral muscle with non-absorbable stay suture. Measured ventricular lead $R$ wave, the threshold, and lead impedance were $12 \mathrm{mV}$, $0.4 \mathrm{~V}$, and $650 \mathrm{ohms}$, respectively. Postoperative fluoroscopic images confirmed the satisfactory positioning of ventricular lead. The pacemaker implantation procedure was uneventful. The patient was discharged after 3 days.

The patient was again admitted with one episode of syncope and twitching in the right upper quadrant of the abdomen. Electrocardiogram showed atrial fibrillation with a ventricular rate of $36 /$ minute. A pulsation in her abdomen due to diaphragmatic stimulation was observed. Temporary pacemaker lead was inserted through the right femoral route and connected to the pulse generator. Fluoroscopy showed twisted ventricular

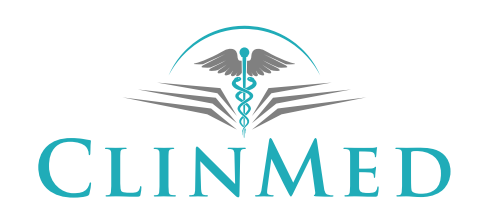

INTERNATIONAL LIBRARY 


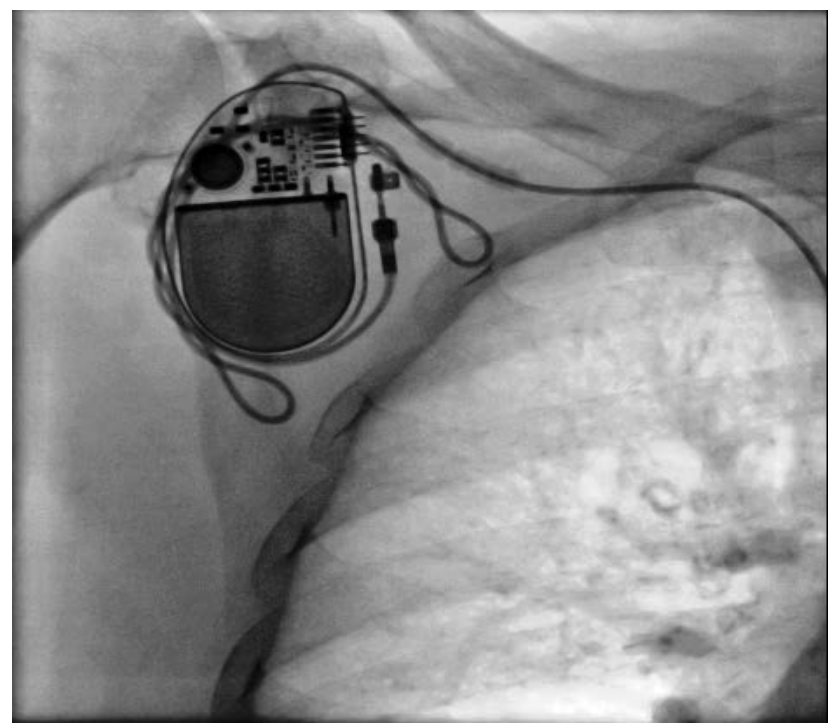

Figure 1: Fluoroscopy showing twisted ventricular lead.

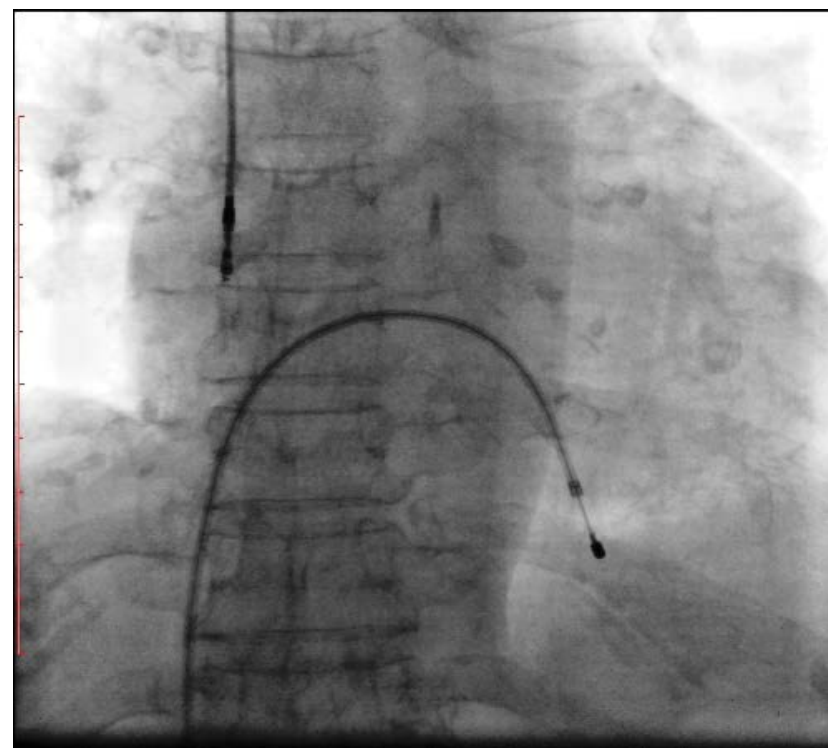

Figure 2: Fluoroscopy showing retracted ventricular lead into the right atrium.

lead and its retraction into the right atrium (Figure 1 and Figure 2). A diagnosis of pacemaker Twiddler's syndrome was made. Under all aseptic precautions, the pacemaker pocket was immediately reopened. The condition of the lead just after opening the pocket and after uncoiling is shown (Figure 3 and Figure 4). A new active fixation screwing ventricular lead was inserted at the right ventricular apex. The parameters were checked, $R$ wave, the threshold, and lead impedance were $14 \mathrm{mV}, 0.5 \mathrm{~V}$, and 700 ohms, respectively. The pulse-generator was fixed on the pectoral muscle with non-absorbable suture. Postoperative hospital stay was uneventful, and the patient was asymptomatic at subsequent follow-ups.

\section{Discussion}

Twiddler syndrome was originally described with pacemakers; the condition has also been reported with implantable cardioverter-defibrillators. Various case reports have been published in the past describing this

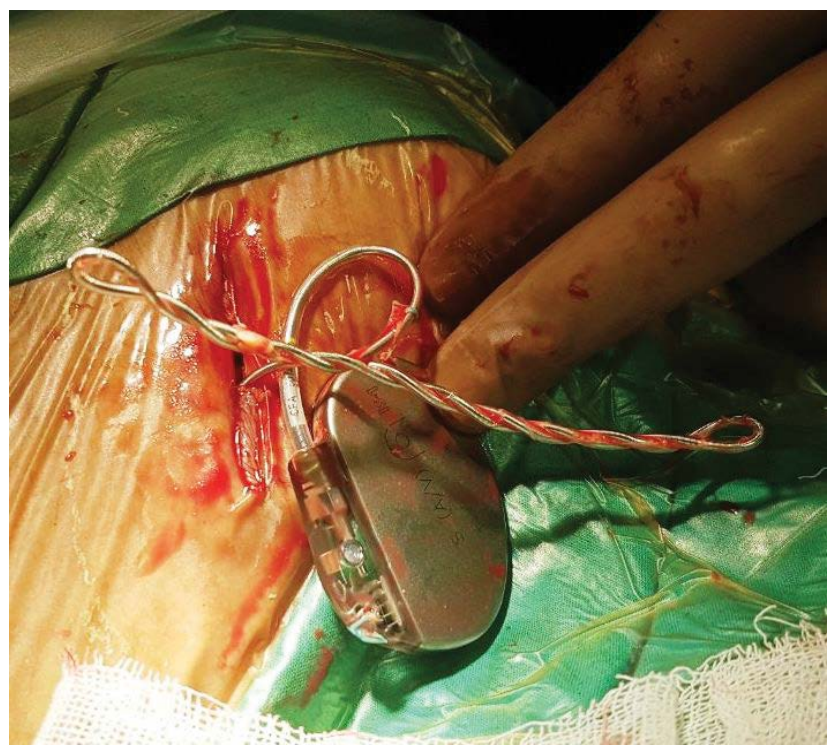

Figure 3: The condition of the ventricular lead after opening the pacemaker pocket. The lead is twisted multiple times on itself.

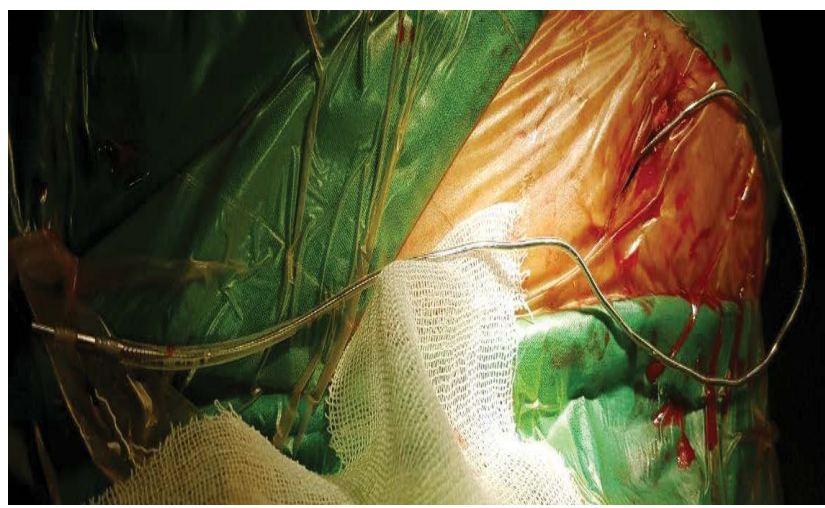

Figure 4: The condition of the lead after uncoiling.

syndrome. The reported incidence of Twiddler's syndrome is around $0.07-7 \%[2,3]$. This complication usually occurs in the first year of implantation, it may occur after one year [4]. The earliest reported case was at 17 hours after implantation [2]. Our patient presented after 4 weeks. It is of particular importance in pacemaker-dependent patients. It is dangerous in patients with a defibrillator, because of inappropriate shocks due to the loss of adequate sensing and capture and possible false treatment of malignant ventricular arrhythmias. Elderly age group, pediatric age, obesity, female gender, psychiatric illness, cognitive dysfunction and the large pocket size relative to the device size are the risk factors for this condition [5]. In almost all cases of Twiddler Syndrome, there is a history of manipulation, by the patient. Various mechanisms have been proposed. It is assumed that the action of scratching, acting on predisposing factors (tissue laxity, large pockets etc.), leads to the rotation of the device body with stretching and dislodgement of the leads which probably rewind on their own [6]. Another mechanism is based on the fact that scratching involves a stretching of leads which are so displaced and rewound on themselves [6]. 
Patient with pacemaker Twiddler syndrome either present with syncope, due to symptomatic bradycardia or with diaphragmatic pacing as a result of phrenic nerve stimulation. Rhythmic arm twitching due to stimulation of brachial plexus has also been reported [7]. Chest X-ray/fluoroscopy and ECG are simple and easily available investigations for its diagnosis. Once the diagnosis is made, a temporary pacemaker is inserted and the patient is shifted to the catheterization laboratory for a repeat procedure. The lead, if damaged is to be replaced. Repeat procedure, hospital stays and a new lead, if required increases the cost. This is of considerable importance in developing countries. To prevent twiddler's it is very important to identify the patients at risk. Minimizing the pocket size, and suture fixation of the pulse generator can prevent the occurrence of Twiddler's syndrome. Some authors have also suggested the use of a Dacron patch, to promote tissue growth around the device [8]. It is also important to educate the patient and family about living with a pacemaker and similar devices.

\section{Conclusion}

Pacemaker Twiddler's syndrome should always be considered as a cause of pacemaker failure in an elderly patient presenting with bradyarrhythmias following pacemaker implantation. Electrocardiogram and chest $\mathrm{X}$-ray are simple and easily-available first-line investigations for its diagnosis. Readmission and lead repositioning are always required. This increases the cost especially when the lead is damaged. Proper patient education and counseling against further manipulation are very important to long-term management.

\section{Informed Consent}

Written informed consent was obtained from the patient for publication of this report and any accompanying images.

\section{Conflicts of interest}

None.

\section{References}

1. Bayliss CE, Beanlands DS, Baird RJ (1968) The pacemakertwiddler's syndrome: A new complication of implantable transvenous pacemakers. Can Med Assoc J 99: 371-373.

2. Fahraeus T, Hijer CJ (2003) Early pacemaker Twiddler syndrome. Europace 5: 279-281.

3. Hill PE (1987) Complications of permanent transvenous cardiac pacing: A 14-year review of all transvenous pacemakers inserted at one community hospital. Pacing Clin Electrophysiol 10: 564-570.

4. Dursun I, Yesildag O, Soylu K, Yilmaz O, Yasar E, et al. (2006) Late pacemaker Twiddler syndrome. Clin Res Cardiol 95: 547-549.

5. Mandal S, Pande A, Kahali D (2012) A Rare Case of Very Early Pacemaker Twiddler's Syndrome. Heart Views 13: 114-115.

6. Casale M, Imbalzano E, Dattilo G (2014) Twiddler Syndrome: A Rare Complication with Important Clinical Relevance. Journal of Cardiology and Therapy 1: 108-110.

7. Nicholson WJ, Tuohy KA, Tilkemeier P (2003) Twiddler's syndrome. N Engl J Med 348: 1726-1727.

8. Furman S (1995) Defibrillator Twiddler's syndrome. Ann Thorac Surg 59: 544-546. 